\title{
Highly insulating ferromagnetic cobaltite heterostructures
}

\author{
Woo Seok Choi ${ }^{1,2}$, Kyeong Tae Kang ${ }^{1}$, Hyoungjeen Jeen ${ }^{2,3}$, Zheng Gai ${ }^{4}$, Ho Nyung Lee² \\ 1Department of Physics, Sungkyunkwan University, Suwon 16419, Korea \\ 2Materials Science and Technology Division, Oak Ridge National Laboratory, Oak Ridge TN 37831, USA \\ 32Department of Physics, Pusan National University, Busan 46241, Korea \\ ${ }^{4}$ Center for Nanophase Materials Sciences, Oak Ridge National Laboratory, Oak Ridge TN 37831, USA \\ *e-mail: choiws@skku.edu
}

Ferromagnetic insulators are rather rare but possess great technological potential in, for example, spintronics. Individual control of ferromagnetic properties and electronic transport provides a useful design concept of multifunctional oxide heterostructures. We studied the close correlation among the magnetism, atomic structure, and electronic structure of oxide heterostructures composed of the ferromagnetic perovskite $\mathrm{LaCoO}_{3}$ and the antiferromagnetic brownmillerite $\mathrm{SrCoO}_{2.5}$ epitaxial thin film layers. By reversing the stacking sequence of the two layers, we could individually modify the electric resistance and saturation magnetic moment. The ferromagnetic insulating behavior in the heterostructures was understood in terms of the electronic reconstruction at the oxide surface/interfaces and crystalline quality of the constituent layers. 


\section{Introduction}

Ferromagnetic exchange interactions in transition metal oxides have been interpreted predominantly as double exchange or Stoner type [1-4]. In perovskite manganites, for example, double exchange occurs between two magnetic manganese ions linked by an oxygen ion. Electron hopping is considered between the two neighboring sites, and it proceeds without spin-flip of the hopping electron. Therefore, metallic conduction is inevitable in a conventional double exchange scheme as well as in itinerant ferromagnetism, which explains why ferromagnetic insulators are not very common.

Although quite rare, ferromagnetic insulating transition metal oxides do exist, and they possess great potential for spintronic applications, including spin valves [5-13]. For example, ferromagnetic insulators can serve as spin filters, while simultaneously acting as a tunneling barrier, within a metal-insulator-metal heterostructure. To properly employ the ferromagnetic insulating property, however, independent control of the electronic and magnetic characteristics is required. In particular, it would be interesting to search for ways to decouple the ferromagnetic property from the metallic behavior so as to realize magnetic heterostructure devices based on transition metal oxides.

Both lanthanum and strontium cobalt oxides are great platforms for the study of magnetism, because they exhibit intriguing spin state and ordering resulting from the coupling among charge, spin, lattice and orbital. While bulk $\mathrm{LaCoO}_{3}$ reveals an antiferromagnetic ordering with spin state transition from low-spin (LS) to high-spin (HS) states as temperature decreases [14, 15], epitaxial $\mathrm{LaCoO}_{3}$ thin films under tensile strain exhibit a robust ferromagnetic long-range ordering with an insulating behavior [16-18]. Microscopic and spectroscopic studies have shown a close correlation between the strain state and spin ordering in $\mathrm{LaCoO}_{3}$ thin films [19-26]. On the other hand, $\mathrm{SrCoO}_{x}$ undergoes a topotactic phase transformation from a ferromagnetic metallic phase $(x$ $=3.0$, perovskite) to an antiferromagnetic insulating phase ( $x=2.5$, brownmillerite) [27-29]. The large deviation in oxygen stoichiometry modifies the local electronic environment of the Co ions and their valence states from $4+$ to $3+[30]$. Such diversity in magnetic properties and the compatibility of the building blocks allow us to examine cobaltite heterostructures as a candidate for tunable ferromagnetic insulators. 
In this work, we studied structural, electrical, and magnetic properties of oxide heterostructures composed of $\mathrm{LaCoO}_{3}$ (LCO) and $\mathrm{SrCoO}_{2.5}$ (SCO) to show that metallic and ferromagnetic behaviors can be tuned by heterostructuring. In particular, we changed the stacking sequence between $\mathrm{LCO}$ and $\mathrm{SCO}$ on (001) $\mathrm{SrTiO}_{3}$ (STO) substrates, to control the ferromagnetic insulating characteristics in the resulting heterostructures. While both $\mathrm{LCO} / \mathrm{SCO} / / \mathrm{STO}$ and $\mathrm{SCO} / \mathrm{LCO} / / \mathrm{STO}$ heterostructures exhibit a robust ferromagnetic and insulating behavior, the SCO/LCO//STO heterostructure shows a higher magnetization as well as a higher resistivity compared with the $\mathrm{LCO} / \mathrm{SCO} / \mathrm{STO}$ heterostructure. This result contradicts the conventional double exchange model of ferromagnetism in transition metal oxides, even more so because both heterostructures consist of exactly the same constituent layers with the same degree of epitaxial strain. We propose that the stacking sequence and resultant modifications at the interface, possibly based on electronic reconstruction at a polar/nonpolar interface, are responsible for the modified ferromagnetic insulating characteristic in cobaltite heterostructures.

\section{Experimental methods}

The high-quality epitaxial LCO and SCO thin films were grown on atomically flat single crystalline STO (001) substrates using pulsed laser epitaxy at $750^{\circ} \mathrm{C}[23,30]$. A $\mathrm{KrF}$ excimer laser $(\lambda=248 \mathrm{~nm})$ with a laser fluence of $1.0 \mathrm{~J} \mathrm{~cm}^{-2}$ at a repetition rate of $10 \mathrm{~Hz}$ was used. The oxygen partial pressure during the growth was 100 mTorr. The crystal structure and epitaxial relations of the thin films were characterized using high-resolution X-ray diffraction (XRD). The thickness of each layer was $5 \pm 1 \mathrm{~nm}$, as determined by X-ray reflectometry.

The resistivity as a function of temperature $\rho(T)$ was measured using a physical property measurement system using the Van der Pauw geometry with In electrodes and Au wires. The magnetization as functions of temperature $M(T)$ and magnetic field $M(H)$ was measured using a superconducting quantum interference device.

\section{Results and discussions}

In order to examine the coupling between the ferromagnetic and insulating properties in cobaltite heterostructures, we designed two heterostructures using ferromagnetic LCO and antiferromagnetic SCO, as schematically shown in Fig. 1. Figure 1(a) (Figure 1(b)) shows the $\mathrm{LCO} / \mathrm{SCO} / \mathrm{STO}$ (SCO/LCO//STO) heterostructure, where the SCO (LCO) layer was first 
deposited on STO, followed by the LCO (SCO) layer deposition. Note that it is not trivial to predict whether the octahedral $\mathrm{CoO}_{2}$ layer or the tetrahedral $\mathrm{CoO}$ layer will be first deposited on top of the surface. In the schematic, we follow a recent observation by Meyer et al., where they confirmed that the tetrahedral $\mathrm{CoO}$ layer comes first on top of the $\mathrm{TiO}_{2}$-terminated STO surface based on scanning transmission electron microscopy [31]. Another point worth mentioning is the difference in the $A$-site and $B$-site networks within the perovskite $A B \mathrm{O}_{x}$ framework of the heterostructures. For LCO/SCO//STO, the $A$-site network is not disturbed at the SCO//STO interface. Furthermore, only the $A$-site network is disturbed at the $\mathrm{LCO} / \mathrm{SCO}$ interface, preserving the $B$-site network. On the contrary, for $\mathrm{SCO} / \mathrm{LCO} / / \mathrm{STO}$, both $A$-site and $B$-site networks are discontinuous at the LCO//STO interface.

Based on the discussion regarding the atomic layers sequence, further differences between the heterostructures can be implied regarding the valence states of Co. The nominal valence states of Co ions are $3+$ for both the LCO and SCO layers. However, we could consider a charge transfer near the interface between polar (LCO) and nonpolar (STO) oxides, which might result in the polar catastrophe. It should be noted that LCO thin films on STO substrates reveal an insulating behavior [32]. Therefore, we can conclude that the Ti valence state in LCO//STO does not change (from 4+ to 3.5+) as efficiently as in the prominent case of a $\mathrm{LaAlO}_{3}$ thin film on a STO substrate $[33,34]$. Instead, we might expect an increase in the Co valence state from $3+$ to $3.5+$ at the interface although $\mathrm{Co}^{4+}$ is rather unstable $[35,36]$. If this were the case, $\mathrm{Co}^{4+}$ within the perovskite framework with $\mathrm{Sr}$ as the $A$-site cation could further stabilize the ferromagnetism in the heterostructure as in ferromagnetic $\mathrm{SrCoO}_{3-\delta}$.

Figure 2 manifests the high-quality crystalline structure of our heterostructures, characterized by XRD. LCO has a pseudocubic lattice constant $a_{\mathrm{c}}=3.8029 \AA$ and experiences tensile strain when grown on STO ( $\mathrm{a}=3.905 \AA)$ [23]. On the other hand, SCO has pseudo-tetragonal lattice constants $a_{\mathrm{t}}=3.905$ and $c_{\mathrm{t}} / 2=3.936 \AA$, and therefore, has a larger out-of-plane lattice compared with STO [30]. This is clearly observed in the XRD results of the single-phase thin films $(\sim 40 \mathrm{~nm})$ as shown in Figs. 2(a) and 2(b). Compared with LCO, the SCO thin film shows additional halforder peaks in-between the perovskite peaks, indicating the presence of ordered oxygen vacancies in the brownmillerite structure, as mentioned in the discussion of Fig. 1 [37]. The magnified 
region in Fig. 2(b) also indicates the high quality of the film with Laue fringes originating from the sharp interfaces and surfaces.

Based on the phase-pure crystalline thin films of LCO and SCO, we constructed heterostructures by stacking each layer with a 5-nm thickness in reverse sequences. The low angle $\mathrm{x}$-ray reflectometry in Fig. 2(c) shows that the total thicknesses of the heterostructure films were close to the intended thickness of $\sim 10 \mathrm{~nm}$. In addition, both of the heterostructure films showed the expected peak features, from both the LCO and SCO layers, as shown in Fig. 2(d). Both heterostructure films were also epitaxially strained revealing the coherent lattice with the substrate one, without any lattice relaxation as evidenced in the reciprocal space maps shown in Figs. 2(f) and 2(g).

However, we also observed major structural differences between the two heterostructures. First, the half-order peaks from SCO were more pronounced for LCO/SCO//STO compared with that for SCO/LCO//STO. On the other hand, the intensity of the LCO peak was larger for $\mathrm{SCO} / \mathrm{LCO} / \mathrm{STO}$. The full width at half maximum (FWHM) of the rocking curve peaks for the LCO layer showed a similar trend. In particular, the FWHM values were much larger for $\mathrm{LCO} / \mathrm{SCO} / / \mathrm{STO}\left(\sim 0.14^{\circ}\right)$ compared with that of the SCO/LCO//STO heterostructure $\left(\sim 0.05^{\circ}\right)$. These differences indicated that the layer in direct contact with the STO substrate has better crystallinity than the layer above. This result is natural, because the roughness of the cobaltite thin film surfaces for both LCO and SCO are generally higher than that for the STO surface.

Both of the cobaltite heterostructures studied here, i.e., LCO/SCO//STO and SCO/LCO//STO, showed a robust ferromagnetic ordering. The epitaxial LCO thin film on STO showed ferromagnetic characteristics with a Curie temperature of $\sim 80 \mathrm{~K}[18,23]$. On the other hand, the brownmillerite SCO thin film is known to be antiferromagnetic [30]. Therefore, we expected magnetic moment coming from the LCO layer only, and thus, normalized magnetization by the number of Co ions in the LCO layers (solid lines). We also show the normalized magnetization by the number of total Co ions in the heterostructure (dashed lines) for comparison. Indeed, we observed a clear ferromagnetic transition at $\sim 80 \mathrm{~K}$ from the $M(T)$ curves (Fig. 3(a)) for both of the heterostructure thin films, suggesting that the magnetic properties mostly originate from the LCO layer in the heterostructures, as expected. 
Ideally, the electric and magnetic properties of the two heterostructures should be the same. However, partly because of the structural discrepancy and interface effect mentioned previously, we found discrepancies in the magnetic characteristics between the two heterostructure thin films. Despite the same number of magnetic Co ions, we observed an overall higher saturation magnetic moment for SCO/LCO//STO compared with LCO/SCO//STO in both the $M(T)$ and $M(H)$ curves. The high magnetization might originate from the better alignment of the spins because of the superior crystal quality of LCO in the SCO/LCO//STO heterostructure thin film compared with LCO/SCO//STO. In addition, the saturation magnetic moment for the SCO/LCO//STO heterostructure was yet larger compared to the LCO thin film on STO (grey line) in $M(H)$ curves. This implies that additional Co ions in the SCO layer might further contribute to the ferromagnetism via the proximity effect. Because the SCO layer can be considered to be grown on a ferromagnetic substrate (LCO) for the SCO/LCO//STO heterostructure, the antiferromagnetically aligned spins might have been influenced by the Co spins in the LCO layer. Such an effect is apparently suppressed in the LCO/SCO//STO heterostructure, where the $\mathrm{LCO} / \mathrm{SCO}$ interface might not be as atomically sharp as the LCO//STO interface in the $\mathrm{SCO} / \mathrm{LCO} / / \mathrm{STO}$ heterostructure. It should also be noted that the magnetic coercive field $H_{\mathrm{c}}$ in the $M(H)$ curves was higher for LCO/SCO//STO ( 0.66 T) compared with that for SCO/LCO//STO $(\sim 0.49 \mathrm{~T})$. Note that $H_{\mathrm{c}}$ for the single-phase LCO film on STO was $\sim 0.3 \mathrm{~T}$. The higher $H_{\mathrm{c}}$ in the heterostructure might originate from partial pinning of the magnetic moment in the LCO layer to the magnetic moment in the adjacent SCO layer. Yet, $H_{\mathrm{c}}$ is still higher in LCO/SCO//STO than that in SCO/LCO//STO, possibly caused by the higher defect concentration in the LCO layer.

Together with the robust ferromagnetic ordering, the heterostructures were highly insulating. Moreover, the sample with higher magnetization actually showed higher electric resistivity, which is counter intuitive based on the general double exchange mechanism in transition metal oxides. The SCO/LCO//STO heterostructure film showed a robust insulating behavior of increasing resistivity with decreasing temperature. The resistivity increased over four orders of magnitude from room temperature to $\sim 50 \mathrm{~K}$. On the other hand, the LCO/SCO//STO heterostructure reveal a lower resistivity while preserving the insulating-temperature-dependent behavior. Note that the resistivity value of LCO/SCO//STO was comparable to that of the LCO thin film on STO substrate [32]. The activation energy calculated based on the Arrhenius 
equation (inset of Fig. 4) also showed a higher value for the SCO/LCO//STO heterostructure $(0.247 \mathrm{eV})$, compared to that for LCO/SCO//STO heterostructure $(0.160 \mathrm{eV})$. These values are comparable with the optical band gap of $\mathrm{LCO}(\geq 0.1 \mathrm{eV})[38]$.

It should be noted that the brownmillerite SCO thin films usually show a more insulating property (higher resistivity) compared with the LCO thin films. The in-plane (parallel) geometry of the resistivity measurement, thus, suggested that the measured transport properties of the heterostructures are mostly coming from the less insulating LCO layer. The LCO/SCO//STO heterostructure exhibited similar resistivity values as the LCO//STO thin films, because they share the same electronic geometry. In particular, they both have a polar-nonpolar interface and a surface with air. The two thin films should undergo the same kind of electronic reconstruction, if required, to prevent the polar catastrophe. On the other hand, the LCO layer within the SCO/LCO//STO heterostructure does not contact air, and there is an additional multivalent SCO layer which might act as a charge reservoir. This reservoir is now in contact with air, which could by itself subject to electronic reconstruction. The possible electronic reconstruction of the SCO layer might be another reason for the higher saturation magnetic moment in the SCO/LCO//STO heterostructure, i.e., by changing the valence state of Co in the topmost SCO layer.

\section{Conclusion}

In conclusion, we studied the physical properties of cobaltite heterostructures. Because of the reversed stacking sequence of the ferromagnetic perovskite $\mathrm{LaCoO}_{3}$ and the antiferromagnetic brownmillerite $\mathrm{SrCoO}_{2.5}$, a more insulating heterostructure with higher magnetic moment was obtained in the $\mathrm{SrCoO}_{2.5} / \mathrm{LaCoO}_{3} / \mathrm{SrTiO}_{3}$ heterostructure. This observation is in contrast to the common double exchange scheme. Such tunability of the ferromagnetic insulating behavior could be attributed to the crystalline quality of each layer and reconstruction of ionic interfaces between the constituent layers.

\section{Acknowledgements}

This work was supported by the U.S. Department of Energy, Office of Science, Basic Energy Sciences, Materials Sciences and Engineering Division. The data analysis on the physical property measurements was supported by Basic Science Research Programs through the National 
Research Foundation of Korea (NRF-2014R1A2A2A01006478). The magnetic measurement was conducted at the Center for Nanophase Materials Sciences, which is a DOE Office of Science User Facility.

\section{Figure Captions}

Figure 1 | Schematic diagrams of cobaltite heterostructures seen along the [110] direction of the STO substrate. (a) LCO/SCO//STO heterostructure. For the SCO//STO interface, only the $B$-site network is disturbed ( $\mathrm{Ti} \rightarrow \mathrm{Co}$ ), while the $A$-site network $(\mathrm{Sr})$ is not disturbed. On the other hand, for the $\mathrm{LCO} / \mathrm{SCO}$ interface, only the $A$-site network is disturbed $(\mathrm{Sr} \rightarrow \mathrm{La}$ ), while the $B$-site network (Co) is not disturbed. (b) SCO/LCO//STO heterostructure. For the LCO//STO interface, both $A$-site and $B$-site networks are disturbed ( $\mathrm{Sr} \rightarrow \mathrm{La}$ and $\mathrm{Ti} \rightarrow \mathrm{Co}$, respectively). On the other hand, for the SCO/LCO interface, only the $A$-site network is disturbed ( $\mathrm{La} \rightarrow \mathrm{Sr}$ ), while the $B$-site network (Co) is not disturbed. All Co ions are considered to have nominal 3+ valence state.

Figure 2 | Structural characterization of LCO/SCO heterostructures. (a) XRD $\theta-2 \theta$ scans of LCO and SCO single-layer thin films. The region in the dotted box is magnified in (b). (d) XRD $\theta-2 \theta$ scans for the $\mathrm{LCO} / \mathrm{SCO}$ and SCO/LCO heterostructures. The regions in the dotted boxes are magnified in (c) and (e). Both peaks from the LCO and SCO layers can be clearly observed. The asterisks denote the substrate peaks. XRD reciprocal space maps of (f) LCO/SCO and (g) SCO/LCO heterostructures around the (114) Bragg reflection of the STO substrate. These maps show coherently strained films without any lattice relaxation.

Figure 3 | Robust ferromagnetic properties. Magnetization of LCO/SCO heterostructures as functions of (a) temperature, $M(T)$, and (b) magnetic field, $M(H) . M(T)$ and $M(H)$ were measured at $1,000 \mathrm{Oe}$ and $5 \mathrm{~K}$ respectively. $\mathrm{SCO} / \mathrm{LCO} / \mathrm{STO}$ (blue) shows a larger magnetic response compared with LCO/SCO//STO (red). The solid red and blue curves represent the normalized magnetization considering that only Co ions in $\mathrm{LCO}(5 \mathrm{~nm})$ are responsible for the magnetization. The dashed red and blue curves represent the normalized magnetization considering that all of the Co ions in the heterostructure $(10 \mathrm{~nm})$ are aligned. The dotted grey curve shows $M(T)$ and $M(H)$ of a LCO thin film for comparison. 
Figure 4 | Transport properties. Temperature-dependent resistivity curves of the heterostructures. Both heterostructures show a highly insulating behavior. SCO/LCO show a higher resistivity than that of the $\mathrm{LCO} / \mathrm{SCO}$ heterostructure for the entire temperature range.

\section{References}

[1] G.H. Jonker, J.H. Van Santen, Ferromagnetic compounds of manganese with perovskite structure, Physica, 16 (1950) 337-349.

[2] C. Zener, Interaction Between the $d$ Shells in the Transition Metals, Phys. Rev., 81 (1951) 440-444.

[3] P.W. Anderson, H. Hasegawa, Considerations on Double Exchange, Phys. Rev., 100 (1955) $675-681$.

[4] Y.-J. Song, K.-W. Lee, Effects of magnetovolume and spin-orbit coupling in the ferromagnetic cubic perovskite $\mathrm{BaRuO}_{3}$, J. Korean Phys. Soc., 62 (2013) 1869-1873.

[5] K. Uchida, J. Xiao, H. Adachi, J. Ohe, S. Takahashi, J. Ieda, T. Ota, Y. Kajiwara, H. Umezawa, H. Kawai, G.E. Bauer, S. Maekawa, E. Saitoh, Spin Seebeck insulator, Nat. Mater., 9 (2010) 894-897.

[6] P. Mahadevan, A. Kumar, D. Choudhury, D.D. Sarma, Charge ordering induced ferromagnetic insulator: $\mathrm{K}_{2} \mathrm{Cr}_{8} \mathrm{O}_{16}$, Phys. Rev. Lett., 104 (2010) 256401.

[7] K. Hasegawa, M. Isobe, T. Yamauchi, H. Ueda, J. Yamaura, H. Gotou, T. Yagi, H. Sato, Y. Ueda, Discovery of ferromagnetic-half-metal-to-insulator transition in $\mathrm{K}_{2} \mathrm{Cr}_{8} \mathrm{O}_{16}$, Phys. Rev. Lett., 103 (2009) 146403.

[8] D.N. Argyriou, J.F. Mitchell, C.D. Potter, D.G. Hinks, J.D. Jorgensen, S.D. Bader, Lattice Effects and Magnetic Order in the Canted Ferromagnetic Insulator $\mathrm{La}_{0.875} \mathrm{Sr}_{0.125} \mathrm{MnO}_{3+\delta}$, Phys. Rev. Lett., 76 (1996) 3826-3829. 
[9] C. Ulrich, G. Khaliullin, S. Okamoto, M. Reehuis, A. Ivanov, H. He, Y. Taguchi, Y. Tokura, B. Keimer, Magnetic order and dynamics in an orbitally degenerate ferromagnetic insulator, Phys. Rev. Lett., 89 (2002) 167202.

[10] Z. Wang, Y. Sun, M. Wu, V. Tiberkevich, A. Slavin, Control of spin waves in a thin film ferromagnetic insulator through interfacial spin scattering, Phys. Rev. Lett., 107 (2011) 146602.

[11] Z. Wang, C. Tang, R. Sachs, Y. Barlas, J. Shi, Proximity-induced ferromagnetism in graphene revealed by the anomalous Hall effect, Phys. Rev. Lett., 114 (2015) 016603.

[12] J.A.C. Bland, T. Taniyama, W.S. Cho, S.J. Steinmueller, Spin selective transport at the ferromagnet/semiconductor interface, Curr. Appl. Phys., 3 (2003) 429-432.

[13] B.G. Kim, J. Dho, S. Jeong, H.B. Shin, H.T. Jo, B.G. Cha, Thickness Dependence of the Perpendicular Magnetic Anisotropy and Magnetic-domain Structures in Nickel-ferrite Films, New Physics: Sae Mulli, 66 (2016) 523-528.

[14] S.R. English, J. Wu, C. Leighton, Thermally excited spin-disorder contribution to the resistivity of $\mathrm{LaCoO}_{3}$, Phys. Rev. B, 65 (2002).

[15] P.M. Raccah, J.B. Goodenough, First-Order Localized-Electron $<->$ Collective-Electron Transition in $\mathrm{LaCoO}_{3}$, Phys. Rev., 155 (1967) 932-943.

[16] D. Fuchs, E. Arac, C. Pinta, S. Schuppler, R. Schneider, H. v. Löhneysen, Tuning the magnetic properties of $\mathrm{LaCoO}_{3}$ thin films by epitaxial strain, Phys. Rev. B, 77 (2008) 014434. [17] J.W. Freeland, J.X. Ma, J. Shi, Ferromagnetic spin-correlations in strained $\mathrm{LaCoO}_{3}$ thin films, Appl. Phys. Lett., 93 (2008) 212501.

[18] D. Fuchs, C. Pinta, T. Schwarz, P. Schweiss, P. Nagel, S. Schuppler, R. Schneider, M. Merz, G. Roth, L. v, ouml, H. hneysen, Ferromagnetic order in epitaxially strained $\mathrm{LaCoO}_{3}$ thin films, Phys. Rev. B, 75 (2007) 144402. 
[19] G.E. Sterbinsky, P.J. Ryan, J.W. Kim, E. Karapetrova, J.X. Ma, J. Shi, J.C. Woicik, Local atomic and electronic structures of epitaxial strained $\mathrm{LaCoO}_{3}$ thin films, Phys. Rev. B, 85 (2012). [20] A. Herklotz, A.D. Rata, L. Schultz, K. Dörr, Reversible strain effect on the magnetization of $\mathrm{LaCoO}_{3}$ films, Phys. Rev. B, 79 (2009) 092409.

[21] V.V. Mehta, N. Biskup, C. Jenkins, E. Arenholz, M. Varela, Y. Suzuki, Long-range ferromagnetic order in $\mathrm{LaCoO}_{3-\delta}$ epitaxial films due to the interplay of epitaxial strain and oxygen vacancy ordering, Phys. Rev. B, 91 (2015) 144418.

[22] S. Park, P. Ryan, E. Karapetrova, J.W. Kim, J.X. Ma, J. Shi, J.W. Freeland, W. Wu, Microscopic evidence of a strain-enhanced ferromagnetic state in $\mathrm{LaCoO}_{3}$ thin films, Appl. Phys. Lett., 95 (2009) 072508-072503.

[23] W.S. Choi, J.-H. Kwon, H. Jeen, J.E. Hamann-Borrero, A. Radi, S. Macke, R. Sutarto, F. He, G.A. Sawatzky, V. Hinkov, M. Kim, H.N. Lee, Strain-Induced Spin States in Atomically Ordered Cobaltites, Nano Lett., 12 (2012) 4966-4970.

[24] C. Hu, K.W. Park, A. Posadas, J.L. Jordan-Sweet, A.A. Demkov, E.T. Yu, Voltagecontrolled ferromagnetism and magnetoresistance in $\mathrm{LaCoO}_{3} / \mathrm{SrTiO}_{3}$ heterostructures, J. Appl. Phys., 114 (2013) 183909.

[25] J.-H. Kwon, W.S. Choi, Y.-K. Kwon, R. Jung, J.-M. Zuo, H.N. Lee, M. Kim, Nanoscale Spin-State Ordering in $\mathrm{LaCoO}_{3}$ Epitaxial Thin Films, Chem. Mater., 26 (2014) 2496-2501. [26] Y. Gao, H. Chang, Q. Wu, Y. Liu, M. Li, F. Liu, H. Zhu, Y. Pang, Y. Yun, Structural transformation, spectroscopic characterization and magnetic properties of $\mathrm{La}_{1-x} \mathrm{Gd}_{x} \mathrm{CoO}_{3}, \mathrm{Curr}$. Appl. Phys., 16 (2016) 922-926.

[27] H. Takei, H. Oda, H. Watanabe, I. Shindo, Growth and properties of strontium cobaltate single crystals, J. Mater. Sci., 13 (1978) 519-522. 
[28] R. Le Toquin, W. Paulus, A. Cousson, C. Prestipino, C. Lamberti, Time-Resolved in Situ Studies of Oxygen Intercalation into $\mathrm{SrCoO}_{2.5}$, Performed by Neutron Diffraction and X-ray Absorption Spectroscopy, J. Am. Chem. Soc., 128 (2006) 13161-13174.

[29] O.T. Tambunan, M.Y. Lee, D.H. Kim, K.J. Parwanta, C.U. Jung, Phase transformation of the brownmillerite $\mathrm{SrCoO}_{2.5}$ thin film through alkaline water electrolysis, J. Korean Phys. Soc., 64 (2014) 1845-1848.

[30] H. Jeen, W.S. Choi, J.W. Freeland, H. Ohta, C.U. Jung, H.N. Lee, Topotactic Phase Transformation of the Brownmillerite $\mathrm{SrCoO}_{2.5}$ to the Perovskite $\mathrm{SrCoO}_{3-\delta}$, Adv. Mater., 25 (2013) 3651-3656.

[31] T.L. Meyer, H. Jeen, X. Gao, J.R. Petrie, M.F. Chisholm, H.N. Lee, Symmetry-Driven Atomic Rearrangement at a Brownmillerite-Perovskite Interface, Adv. Electron. Mater., 2 (2016) n/a-n/a.

[32] J.W. Freeland, J.X. Ma, J. Shi, Ferromagnetic spin-correlations in strained $\mathrm{LaCoO}_{3}$ thin films, Appl. Phys. Lett., 93 (2008) 212501-212503.

[33] N. Nakagawa, H.Y. Hwang, D.A. Muller, Why some interfaces cannot be sharp, Nat. Mater., 5 (2006) 204-209.

[34] W.S. Choi, D.W. Jeong, S.S.A. Seo, Y.S. Lee, T.H. Kim, S.Y. Jang, H.N. Lee, K. MyungWhun, Charge states and magnetic ordering in $\mathrm{LaMnO}_{3} / \mathrm{SrTiO}_{3}$ superlattices, Phys. Rev. B, 83 (2011) 195113.

[35] H. Jeen, W.S. Choi, M.D. Biegalski, C.M. Folkman, I.C. Tung, D.D. Fong, J.W. Freeland, D. Shin, H. Ohta, M.F. Chisholm, H.N. Lee, Reversible redox reactions in an epitaxially stabilized $\mathrm{SrCoO}_{x}$ oxygen sponge, Nat. Mater., 12 (2013) 1057-1063. 
[36] W.S. Choi, H. Jeen, J.H. Lee, S.S.A. Seo, V.R. Cooper, K.M. Rabe, H.N. Lee, Reversal of the Lattice Structure in $\mathrm{SrCoO}_{x}$ Epitaxial Thin Films Studied by Real-Time Optical Spectroscopy and First-Principles Calculations, Phys. Rev. Lett., 111 (2013) 097401.

[37] H. Jeen, Z. Bi, W.S. Choi, M.F. Chisholm, C.A. Bridges, M.P. Paranthaman, H.N. Lee, Orienting Oxygen Vacancies for Fast Catalytic Reaction, Adv. Mater., 25 (2013) 6459-6463. [38] Y. Tokura, Y. Okimoto, S. Yamaguchi, H. Taniguchi, T. Kimura, H. Takagi, Thermally induced insulator-metal transition in $\mathrm{LaCoO}_{3}$ :A view based on the Mott transition, Phys. Rev. B, 58 (1998) R1699. 


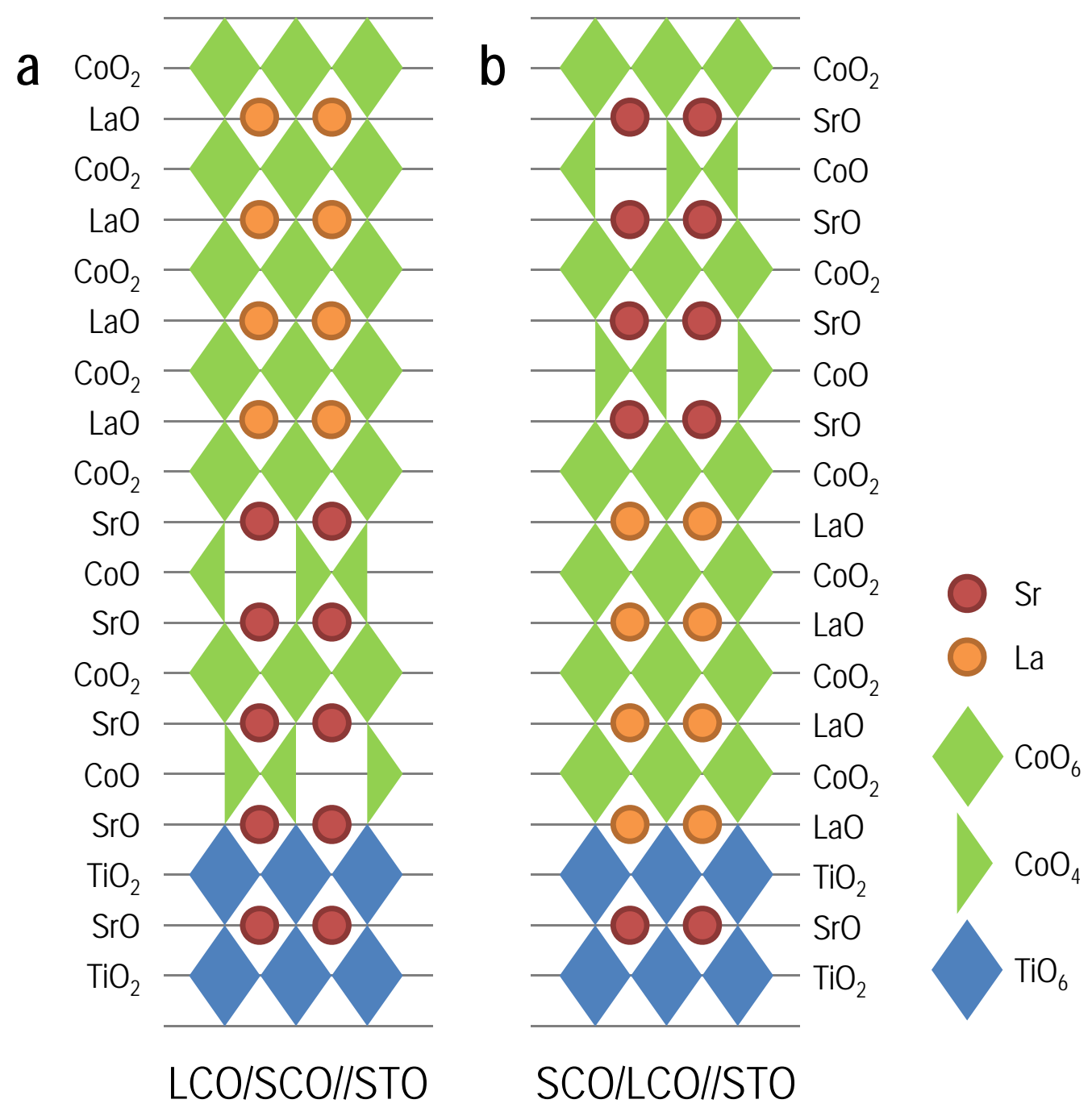



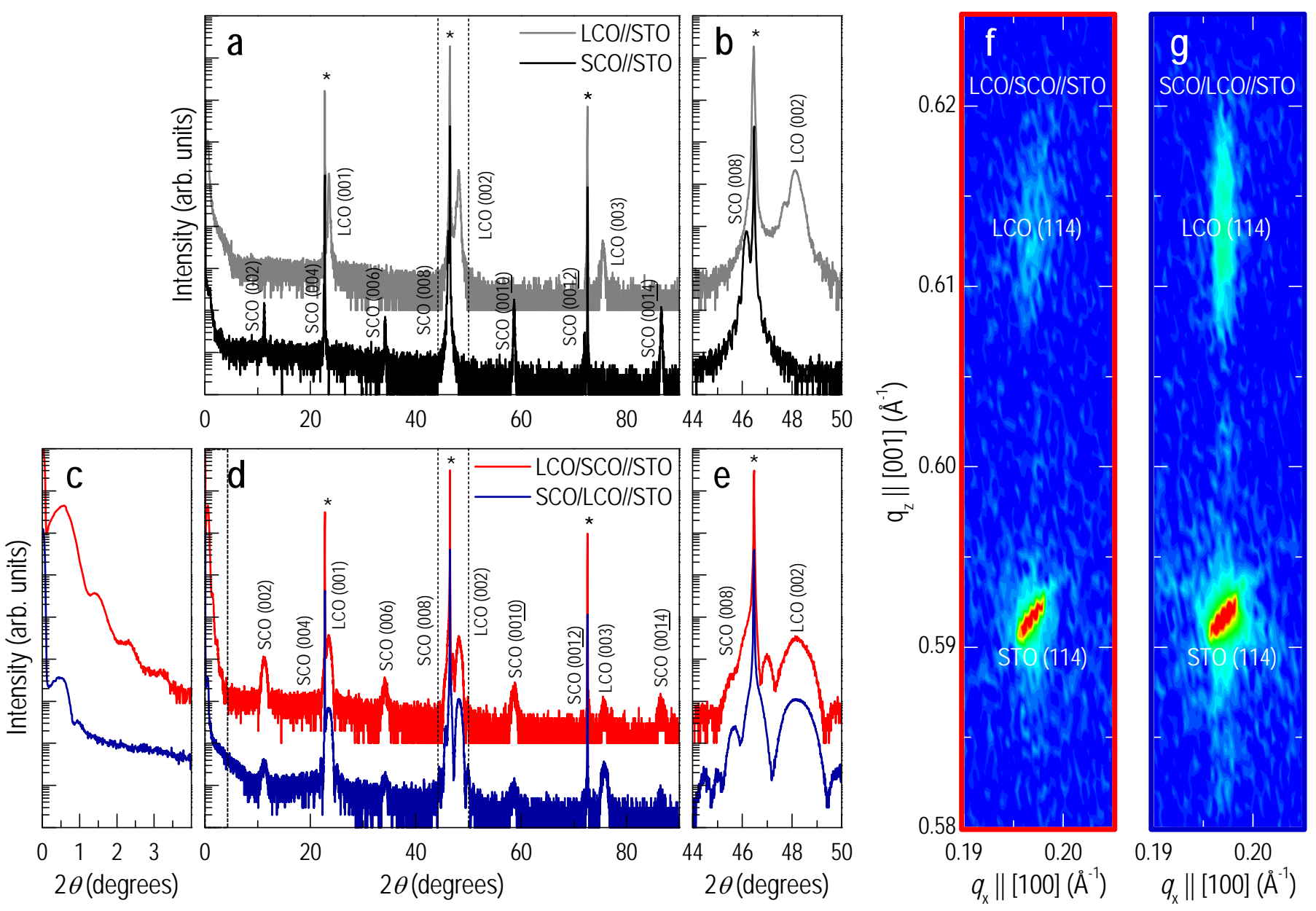

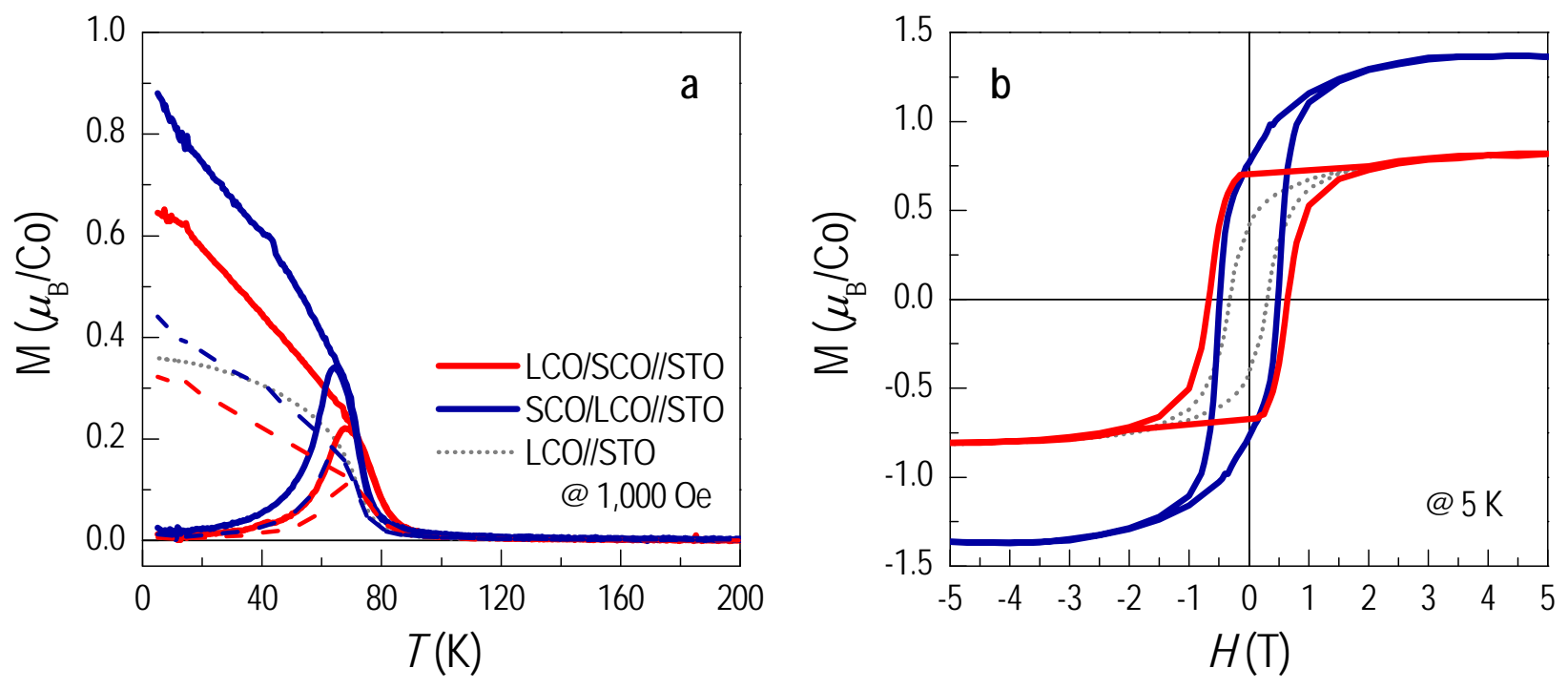


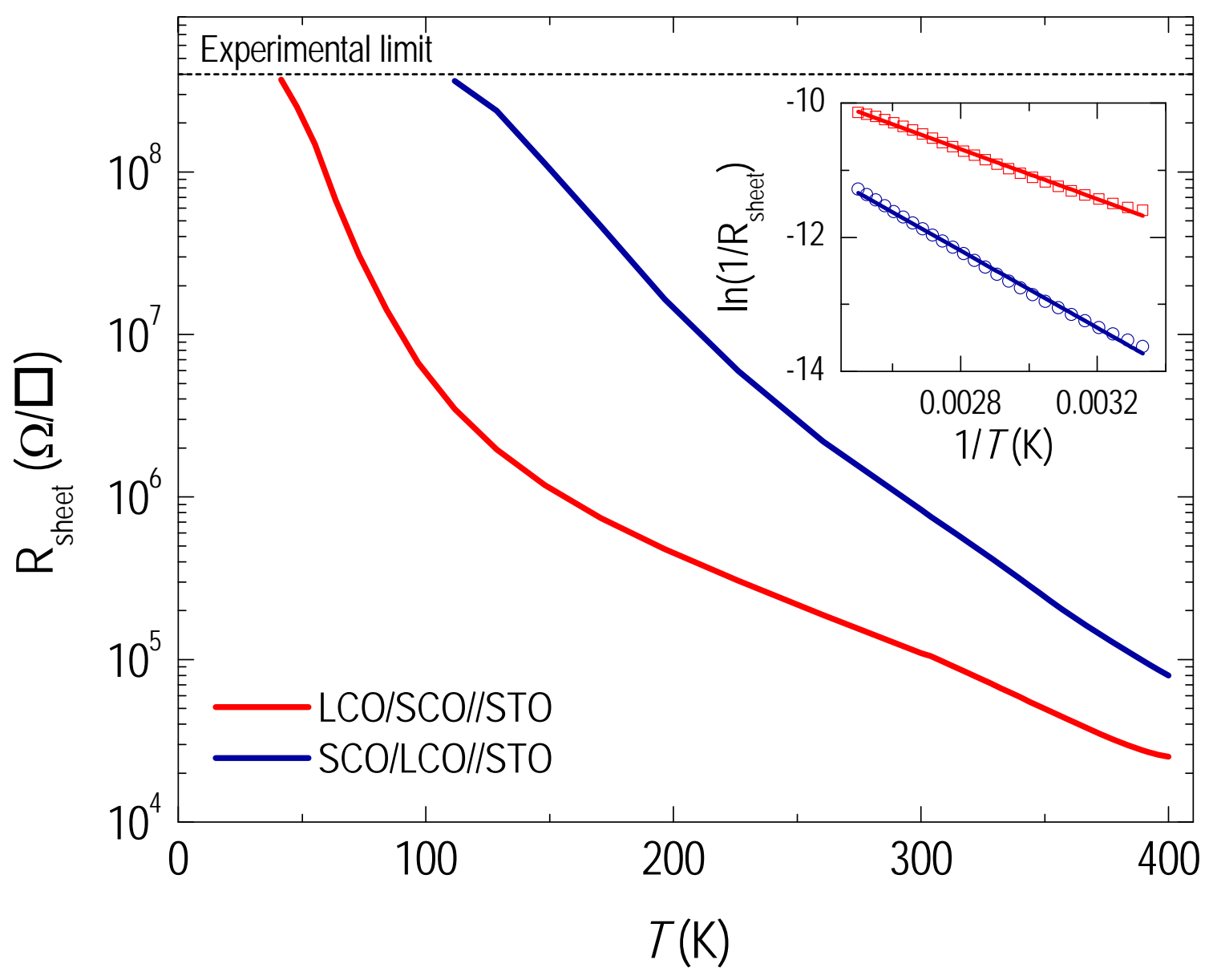

